\title{
Effect of Gravity on Brownian Flocculation
}

\author{
D. H. MELIK ${ }^{1}$ AND H. S. FOGLER \\ Department of Chemical Engineering, The University of Michigan, Ann Arbor, Michigan 48109
}

Received October 7, 1983; accepted February 14, 1984

\begin{abstract}
In previous analyses of simultaneous Brownian and gravity-induced flocculation, it has been assumed that each mechanism contributes independently to the total flocculation rate. In this paper the rigorous problem of coupled Brownian and gravity-induced flocculation is considered for the case of strong Brownian/weak gravity effects. Using a singular perturbation expansion solution of the governing steadystate convective-diffusion equation, coupled flocculation rates are obtained. Comparison of the coupled rates with the rates found from the additivity approach show that additivity is justified only under very restrictive conditions.
\end{abstract}

\section{INTRODUCTION}

In the absence of bulk fluid motion, both Brownian diffusion and differential creaming can cause polydisperse colloidal particles to flocculate if the interparticle interactions are favorable $(1,2)$. In differential creaming the larger particles sweep out the slower moving small particles in their path. A process which is termed sedimentation (gravity-induced) flocculation.

Analysis of the combined effects of Brownian and gravity-induced flocculation is of current research interest. The first work in this area was carried out by Müller (3) who extended Smoluchowski's Brownian theory (4) to account for the polydispersity of particle sizes which gives rise to gravity-induced flocculation. This theory was shown experimentally by Matthews and Rhodes (5) to apply qualitatively for particles in the size range of $0.5-2.0 \mu \mathrm{m}$, but could not give quantitative results since both hydrodynamic interactions and interparticle forces were neglected.

Significant progress has been made in quantitatively describing the deposition of colloidal particles onto various collectors under laminar flow conditions (cf. 6-9, 34, 35). In particular, dispersion, electric double layer,

\footnotetext{
${ }^{1}$ Present address: the Procter \& Gamble Company, Miami Valley Laboratories, Cincinnati, Ohio 45247.
}

and external force fields (e.g., gravity), Brownian diffusion, as well as the hydrodynamic interaction between particles and collectors have been rigorously accounted for. However, these types of flocculation studies are not applicable to the case of two comparably sized colloidal particles since the collector is both stationary and much larger than the particle. Consequently, the effect of mutual particle diffusion and different particle size ratios is absent.

In order to rigorously study the kinetics of simultaneous Brownian and gravity-induced flocculation, it is necessary to solve the convective-diffusion equation which describes the distribution of particles around a reference or test sphere. This equation has been solved in the steady-state for the case of no external force (e.g., gravity, shearing motion, etc.) by Derjaguin and Muller (10), and independently by Spielman (11). Previously Smoluchowski (4) ignored interparticle forces and hydrodynamic interactions; while Fuchs (12) neglected hydrodynamic interactions. The transient case has been investigated by Roebersen and Wiersema (13) and Feke and Prabhu (14). Recently, Van de Ven and Mason (15) and Batchelor (16) have solved the steady-state convectivediffusion equation for the case of strong Brownian motion with weak shearing flows, while Feke and Schowalter (17) have analyzed systems undergoing strong shearing flows with 
weak Brownian diffusion. In this paper a solution of the steady-state convective-diffusion equation is presented for strong Brownian/ weak gravity effects. The case of strong gravity/ negligible Brownian effects has been treated elsewhere (18).

In the analysis given here, only interparticle potentials which result in particle/particle flocculation are considered (i.e., the interparticle potential $V_{\text {int }} \rightarrow-\infty$ as the interparticle separation $H \rightarrow 0$ ). Batchelor $(19,20)$ has solved the steady-state convective-diffusion equation for strong Brownian motion/weak gravity systems when the interparticle potential prohibits particle capture (i.e., $V_{\text {int }} \rightarrow+\infty$ as $H \rightarrow 0$ ). In Batchelor's analysis a regular perturbation solution was adequate; whereas in the present case a solution of the convective-diffusion equation can be found by making use of the perturbation method described by Proudman and Pearson (21) of matching inner and outer expansions.

\section{PHYSICAL MODEL}

A model for the flocculation of dilute suspensions of polydisperse colloidal spheres in quiescent media is described in this section. Because the suspension is assumed to be dilute, only two-particle interactions are included in the analysis.

Consider the interaction between the two unequally sized spheres shown in Fig. 1, where the origin of a Lagrangian coordinate system coincides with the center of the smaller (test or reference) sphere, of radius $a_{1}$. The steadystate single-particle flocculation rate is given by the net inward flux of type- 2 particles, with a radius $a_{2}$, through some surface $A^{*}$ enclosing the test sphere

$$
j_{12}=-\int_{A^{*}}(\mathbf{v} \mathscr{P}) \cdot \mathbf{n} d A,
$$

where $\mathbf{v}=\mathbf{v}(\mathbf{r})$ is the velocity of the second particle relative to the test sphere, and the pair probability density $\mathcal{P}=\mathscr{P}(\mathbf{r})$ describes the number concentration of type- 2 particles in space.

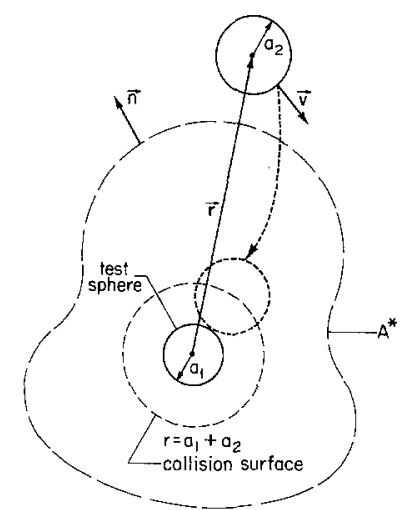

FIG. 1. Schematic for a binary encounter between different sized spheres.

The probability function is governed by a conservation equation for regions of space outside the collision surface which, in steadystate, is given by

$$
\nabla \cdot(\mathbf{v} \mathcal{P})=0, \quad r \geqslant a_{1}+a_{2},
$$

where $r$ is the distance between particle centers. Since colloidal particles are in the low Reynolds number regime, the governing hydrodynamic equations are linear, and the overall flow field can be decomposed into separate contributions (22). For the case of interest, the relative sphere velocity is attributed to three sources: motion due to gravity, motion caused by Brownian diffusion, and motion due to interparticle forces, namely

$$
\mathbf{v}=\mathbf{v}_{\mathrm{g}}+\mathbf{v}_{\mathrm{Br}}+\mathbf{v}_{\text {int }} .
$$

The relative velocity of two spheres in an infinite medium acted upon by gravity can be written as (23)

$$
\begin{aligned}
\mathbf{v}_{\mathrm{g}}= & \left(u_{02}-u_{01}\right) \\
& \times\left\{-\mathcal{A}(R) \cos \theta \mathbf{e}_{\mathrm{r}}+\mathscr{B}(R) \sin \theta \mathbf{e}_{\theta}\right\},
\end{aligned}
$$

where $R=2 r /\left(a_{1}+a_{2}\right)$ is the dimensionless interparticle distance, $\mathcal{A}(R)$ and $\mathcal{B}(R)$ the hydrodynamic corrections to Stokes relative creaming rate $\left(u_{02}-u_{01}\right)$, and $\mathbf{e}_{\mathbf{r}}$ and $\mathbf{e}_{\theta}$ unit vectors in the spherical polar coordinate system (note: gravity acts in the direction $\theta=\pi$ ).

The relative sphere velocity due to Brown- 
ian motion can be formulated in terms of the relative diffusion coefficient and the probability density (24)

$$
\begin{array}{r}
\mathbf{v}_{\mathrm{Br}}=-D_{0}\left\{S(R) \frac{\partial \ln \mathscr{P}}{\partial r} \mathbf{e}_{\mathrm{r}}+\frac{\mathscr{H}(R)}{r} \frac{\partial \ln \mathscr{P}}{\partial \theta} \mathbf{e}_{\theta}\right. \\
\left.+\frac{\mathscr{H}(R)}{r \sin \theta} \frac{\partial \ln \mathcal{P}}{\partial \phi} e_{\phi}\right\},
\end{array}
$$

where $\mathcal{S}(R)$ and $\mathscr{H}(R)$ are the hydrodynamic corrections to the Stokes-Einstein relative diffusion coefficient $D_{0}$, and $\mathbf{e}_{\phi}$ a unit vector in the spherical polar coordinate system.

For spherical particles, interparticle forces only act along line of centers. The relative velocity due to such a force is given by (15)

$$
\mathbf{v}_{\mathrm{int}}=-\frac{g(R) D_{0}}{k T} \frac{\partial V_{\mathrm{int}}}{\partial r} \mathbf{e}_{\mathrm{r}}
$$

where $V_{\text {int }}$ is the total interparticle potential, $k$ the Boltzmann constant, and $T$ the absolute temperature.

Substituting the velocity expressions [4][6] into [2] yields the following steady-state pair-conservation equation:

$$
\begin{aligned}
\frac{\partial}{\partial R} R^{2} \mathcal{S}(R)\left\{\frac{\partial \mathscr{P}}{\partial R}+\frac{\mathcal{P}}{k T} \frac{\partial V_{\text {int }}}{\partial R}\right\} \\
+\mathscr{H}(R)\left\{\frac{\partial^{2} \mathscr{P}}{\partial \theta^{2}}+\frac{\cos \theta}{\sin \theta} \frac{\partial \mathcal{P}}{\partial \theta}+\frac{1}{\sin ^{2} \theta} \frac{\partial^{2} \mathcal{P}}{\partial \phi^{2}}\right\} \\
=\operatorname{Gr}\left\{\cos \theta\left[2 R \mathcal{B}(R) \mathscr{P}-\frac{\partial}{\partial R} R^{2} \mathcal{A}(R) \mathcal{P}\right]\right. \\
\left.+R \mathscr{B}(R) \sin \theta \frac{\partial \mathcal{P}}{\partial \theta}\right\},
\end{aligned}
$$

where the gravity number $G r$ is defined by

$$
\begin{aligned}
G r & =\frac{\left(u_{02}-u_{01}\right)\left(a_{1}+a_{2}\right)}{2 D_{0}} \\
& =\frac{2 \pi g \Delta \rho a_{2}^{4}}{3 k T} \lambda\left(1-\lambda^{2}\right),
\end{aligned}
$$

and represents the relative strength of gravitational to Brownian forces, with $g$ being the local acceleration of gravity, $\Delta \rho$ the density difference between the particles and the sus- pending medium, and $\lambda=a_{1} / a_{2}$ the particle size ratio (note: by definition $0<\lambda \leqslant 1$ ).

In Fig. 2 the gravity number $G r$ is shown as a function of the particle size ratio $\lambda$ for the case $\Delta \rho=0.1 \mathrm{~g} / \mathrm{cm}^{3}$ in a water medium at $T=298^{\circ} \mathrm{K}$ under normal gravity. One notices that the solution of the pair-conservation equation presented in this paper will be valid for particles less than approximately $1.0 \mu \mathrm{m}$ in radius, depending of course on the magnitude of $\Delta \rho, \lambda$, and $g$.

The appropriate steady-state boundary conditions for [7] are

$$
\mathcal{P}=0 \quad \text { at } \quad R=2,
$$

and

$$
P \rightarrow 1 \text { as } R \rightarrow \infty .
$$

Due to the symmetry of gravitational motion the following conditions must hold:

$$
\frac{\partial \mathcal{P}}{\partial \theta}=0 \quad \text { at } \quad \theta=0, \pi
$$

and

$$
\mathscr{P} \neq f(\phi) \quad \text { for all } \phi \text {. }
$$

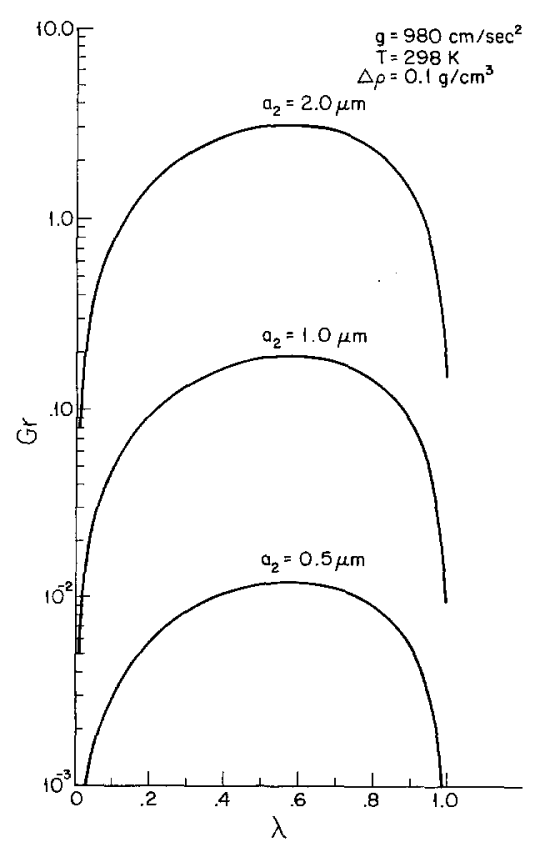

FIG. 2. Effect of the particle size ratio on the gravity number for various particle sizes. 
Equation [12] indicates that gravity does not perturb the particle distribution in the $\phi$ direction.

In the analysis here, the following far-field forms of the hydrodynamic corrections are used (23), which were derived from the farfield mobilities given by Reed and Anderson (25):

$$
\begin{aligned}
& \mathcal{A}(R \rightarrow \infty)=1-\frac{\beta}{R}+0\left(R^{-3}\right), \\
& \mathcal{B}(R \rightarrow \infty)=1-\frac{\beta}{2 R}+0\left(R^{-3}\right), \\
& g(R \rightarrow \infty)=1-\frac{\xi}{R}+0\left(R^{-3}\right),
\end{aligned}
$$

and

$$
\mathscr{H}(R \rightarrow \infty)=1-\frac{\xi}{2 R}+0\left(R^{-3}\right)
$$

where

and

$$
\begin{aligned}
& \beta=\frac{3 \gamma\left(1+\lambda+\lambda^{2}\right)}{(1+\lambda)^{2}}, \\
& \xi=\frac{6 \gamma \lambda}{(1+\lambda)^{2}}
\end{aligned}
$$

$$
\gamma=\frac{(2 / 3)+\left(\mu_{\mathrm{p}} / \mu_{\mathrm{f}}\right)}{1+\left(\mu_{\mathrm{p}} / \mu_{\mathrm{f}}\right)},
$$

with $\mu_{\mathrm{p}}$ being the viscosity of the particles and $\mu_{\mathrm{f}}$ the viscosity of the suspending fluid. For a rigid sphere, the viscosity ratio $\gamma=1$, while for a gas bubble $\gamma=2 / 3$. Complete near-field forms of the hydrodynamic corrections are unavailable; however, as $R \rightarrow 2$ (particle/particle contact),

$$
\begin{aligned}
& \mathcal{A}(R \rightarrow 2)=C_{0}(R-2), \\
& \mathcal{B}(R \rightarrow 2)=C_{1}+\frac{C_{2}}{\ln (R-2)}, \\
& \mathcal{S}(R \rightarrow 2)=C_{3}(R-2),
\end{aligned}
$$

and

$$
\mathscr{H}(R \rightarrow 2)=C_{4}+\frac{C_{5}}{\ln (R-2)},
$$

where $C_{i}$ are constants which depend on both $\lambda$ and $\gamma$. The exact values of these constants are unknown; however this does not affect the analysis presented here since only the behavior of the hydrodynamic corrections as $R \rightarrow 2$ is necessary.

\section{Criteria for a Steady-State Particle Distribution}

In order for a steady-state particle distribution to exist, the net flux of particles crossing the surface $A^{*}$ shown in Fig. 1 must be constant and independent of the choice of $A^{*}$, otherwise regions will exist where there is either an accumulation or depletion of particles. This requirement implies that the two spheres must touch in a finite amount of time. Whether this touching occurs or not depends on the behavior of the interparticle potential $V_{\text {int }}(R)$ as $R \rightarrow 2$. For these very small interparticle distances the dominant contribution to the relative sphere velocity $\mathbf{v}$ will obviously be $\mathbf{v}_{\text {int }}$, and by substituting [22] into [6], one finds that for $(R-2) \ll 1$, the radial component of the relative velocity reduces to

$$
V_{\mathrm{r}}=\frac{d R}{d \tau}=-\frac{C_{3}}{k T}(R-2) \frac{\partial V_{\mathrm{int}}}{\partial R},
$$

where $\tau=4 D_{0} t /\left(a_{1}+a_{2}\right)^{2}$ is the dimensionless time. As pointed out by van de Ven and Mason (26), if

$$
\int_{2}^{2+\omega}\left\{\frac{d R}{(R-2) \frac{\partial V_{\text {int }}}{\partial R}}\right\}
$$

remains finite, where $\omega$ is an arbitrarily small interparticle distance, the two spheres will eventually touch in a finite amount of time. For example, the two particles will touch if $V_{\text {int }}$ follows an inverse power law as described by DLVO theory $(27,28)$.

Therefore, since the interparticle potential vanishes at infinity, i.e.,

$$
\lim _{R \rightarrow \infty} V_{\text {int }}(R)=0,
$$

this requires that

$$
\lim _{R \rightarrow 2} \frac{1}{(R-2) \frac{\partial V_{\mathrm{int}}}{\partial R}}=0,
$$


in order for a collision to occur in a finite amount of time. Equations [25] and [26] are necessary conditions in order for a steady-state to be obtained, although not sufficient conditions.

Even though an interparticle force may lead to eventual contact between the spheres, a steady-state particle distribution may not be possible. In particular, for systems with both a large electrostatic barrier and a secondary minimum (as described by DLVO theory), Feke and Prabhu (14) have shown that the flocculation rate is very time dependent. Initially there is a rapid net inward flux of particles into the secondary minimum with virtually no particles accumulating in the primary minimum. During this induction period the secondary minimum becomes saturated, and "slow" flocculation into the primary minimum ensues. The relaxation time required for this process is highly dependent on the magnitude of the energy barrier.

For potential barriers greater than approximately $17 k T$, the Brownian stability factor is well over $10^{5}(11)$, which is indicative of a fairly stable system (i.e., there is virtually no flocculation occurring in the primary minimum). In this case, secondary minimum (or reversible) flocculation is dominant and a steady-state is not possible even though some sort of quasi-equilibrium may be established. On the other hand, for systems with a potential barrier less than approximately $10 k T$, the relaxation time required for the steady state to be established is only on the order of seconds (29-31, 36). Consequently, in order to ensure that the steady-state assumption is valid, the requirements given by [25] and [26] must hold in addition to a potential barrier of less than approximately $10 k T$.

\section{PERTURBATION ANALYSIS}

To approximate the solution of the steadystate pair-conservation equation for strong Brownian diffusion/weak gravity systems (i.e., $\mathrm{Gr} \ll 1$ ), first consider an expansion of the form:

$$
\mathcal{P}(R, \theta)=\mathscr{P}_{0}+G r \mathcal{P}_{1}+\cdots
$$

Substituting this expansion into [7] yields the following perturbation equations for $\mathcal{P}_{0}$ and $P_{1}$ :

$$
\nabla_{\mathrm{R}}^{2} \mathcal{P}_{0}+\mathscr{H}(R) \nabla_{\theta}^{2} \mathscr{P}_{0}=0
$$

$$
\begin{aligned}
& \nabla_{\mathrm{R}}^{2} \mathscr{P}_{1}+\mathscr{H}(R) \nabla_{\theta}^{2} \mathscr{P}_{1} \\
& =\cos \theta\left[2 R \mathscr{B}(R) \mathscr{P}_{0}-\frac{\partial}{\partial R} R^{2} \mathcal{A}(R) \mathcal{P}_{0}\right] \\
& +R \mathscr{B}(R) \sin \theta \frac{\partial \mathscr{P}_{0}}{\partial \theta}
\end{aligned}
$$

where the operators $\nabla_{\mathrm{R}}^{2}$ and $\nabla_{\theta}^{2}$ are given by:

$$
\begin{aligned}
& \nabla_{\mathrm{R}}^{2} \equiv \frac{\partial}{\partial R} R^{2} \mathcal{S}(R)\left\{\frac{\partial}{\partial R}+\frac{1}{k T} \frac{\partial V_{\mathrm{int}}}{\partial R}\right\}, \\
& \nabla_{\theta}^{2} \equiv \frac{\partial^{2}}{\partial \theta^{2}}+\frac{\cos \theta}{\sin \theta} \frac{\partial}{\partial \theta}
\end{aligned}
$$

with the symmetry condition [12] being used to simplify [31]. The appropriate boundary conditions are

$$
\begin{gathered}
\mathscr{P}_{0}=\mathscr{P}_{1}=0 \quad \text { at } \quad R=2, \\
\mathscr{P}_{0}=1 ; \quad \mathscr{P}_{1}=0 \quad \text { as } \quad R \rightarrow \infty, \\
\frac{\partial \mathscr{P}_{0}}{\partial \theta}=\frac{\partial \mathscr{P}_{1}}{\partial \theta}=0 \quad \text { at } \quad \theta=0, \pi .
\end{gathered}
$$

The zero- and first-order solutions, $\mathscr{P}_{0}$ and $\mathcal{P}_{1}$, are discussed next.

\section{Zero-Order Solution}

The solution to [28], subject to the boundary conditions [32]-[34], is given by $(10,15)$

$$
\mathcal{P}_{0}(R)=e^{-V_{\mathrm{in} /} / k T}\left\{1-\alpha_{\mathrm{Br}} \int_{R}^{\infty} \frac{e^{V_{\mathrm{in} /} / k T}}{G(R) R^{2}} d R\right\}
$$

where

$$
\alpha_{\mathrm{Br}}^{-1}=\int_{2}^{\infty} \frac{e^{V_{\mathrm{in} t / k T}}}{G(R) R^{2}} d R=\frac{W_{\mathrm{Br}}}{2}
$$

with $W_{\mathrm{Br}}$ being the Brownian flocculation stability factor. Making use of [25], one finds that as $R \rightarrow \infty,[35]$ reduces to

$$
\mathcal{P}_{0}(R \rightarrow \infty)=1-\frac{\alpha_{\mathrm{Br}}}{R}
$$


when $\alpha_{\mathrm{Br}} \neq 0$ (note: the case $\alpha_{\mathrm{Br}}=0$ occurs when $V_{\text {int }} \rightarrow+\infty$ as $R \rightarrow 2$ ).

\section{First-Order Solution}

Inner expansion. Since [29] is a linear differential equation, the complete solution is given by the solution of the homogeneous equation

$$
\nabla_{\mathrm{R}}^{2} \mathscr{P}_{1}+\mathscr{H}(R) \nabla_{\theta}^{2} \mathscr{P}_{1}=0
$$

plus a particular solution, one of which is $f_{p}(R)$ $\times \cos \theta$. Substitution of this particular solution into [29] allows one to determine $f_{p}(R)$ from

$$
\begin{aligned}
\nabla_{\mathrm{R}}^{2} f_{p} & -2 \mathscr{H}(R) f_{p} \\
& =2 R \mathcal{B}(R) \mathscr{P}_{0}-\frac{d}{d R} R^{2} \mathcal{A}(R) \mathscr{P}_{0} .
\end{aligned}
$$

Using the separation of variables technique to determine the solution of [38], one finds that the general solution of [29] is given by

$\mathscr{P}_{1}(R, \theta)=f_{p}(R) \cos \theta$

$$
+\sum_{\ell=0}^{\infty} f_{\ell}(R) L_{\ell}(\cos \theta)
$$

where the angular portion of the homogeneous solution $L_{\ell}(\cos \theta)$ is the Legendre polynomial of the first kind, and the radial portion of the homogeneous solution $f_{\ell}(R)$ is determined from (15)

$$
\nabla_{\mathrm{R}}^{2} f_{\ell}-\ell(\ell+1) \mathscr{H}(R) f_{\ell}=0 .
$$

The boundary conditions for [39] and [41] become

$$
f_{i}(R=2)=f_{i}(R=\infty)=0 .
$$

Substituting the near-field asymptotic expressions [20]-[23] into [39], and making use of [26], one finds that for $(R-2) \ll 1$, [39] reduces to

$$
\frac{d}{d R} R^{2}(R-2) \frac{f_{p}}{k T} \frac{\partial V_{\mathrm{int}}}{\partial R}=0 .
$$

Since the particular solution of this equation is just $f_{p}(R \rightarrow 2)=0$, the inner boundary condition of [42] is satisfied.
Making use of the far-field asymptotic relations [13]-[16] and [37], one finds that for large $R[39]$ reduces to

$$
\frac{d}{d R}\left(R^{2} \frac{d f_{p}}{d R}\right)-2 f_{p}=-\alpha_{\mathrm{Br}}
$$

By inspection one can determine that the particular solution $f_{p}$ at large $R$ is

$$
f_{p}(R \rightarrow \infty)=\frac{\alpha_{\mathrm{Br}}}{2} .
$$

This solution, however, does not converge to zero as $R \rightarrow \infty$. The reason for the failure of the classical expansion [27] can be easily explained by the fact that, no matter how small $G r$ is, there is always a region far away from the test sphere where both the diffusion and convection (due to gravity) terms in [7] become of the same order of magnitude. This phenomenon prevents [27] from becoming a uniformly valid approximation to the probability density $\mathscr{P}$. Consequently, the solution given by [40] is only part of an inner expansion valid at small interparticle distances from the test sphere.

Using the far-field solution of [41], namely (15)

$$
f_{\ell}(R \rightarrow \infty)=a_{\ell 1} R^{\ell}+\frac{a_{\ell 2}}{R^{\ell+1}}
$$

where $a_{\ell i}$ are constants of integration, the first order correction for gravity (in the inner region) at large $R$ becomes

$$
\begin{aligned}
& P_{1}(R \rightarrow \infty, \theta)=\frac{\alpha_{\mathrm{Br}}}{2} \cos \theta+a_{01} \\
& +\frac{a_{02}}{R}+\left[a_{11} R+\frac{a_{12}}{R^{2}}\right] \cos \theta+\cdots
\end{aligned}
$$

and the inner expansion at large $R$ is

$$
\begin{aligned}
& \mathcal{P}(R \rightarrow \infty, \theta)=1-\frac{\alpha_{\mathrm{Br}}}{R} \\
& +\operatorname{Gr}\left\{\frac{\alpha_{\mathrm{Br}}}{2} \cos \theta+a_{01}+\frac{a_{02}}{R}\right. \\
& \left.+\left[a_{11} R+\frac{a_{12}}{R^{2}}\right] \cos \theta+\cdots\right\}+\cdots
\end{aligned}
$$


The constants $a_{\ell i}$ are determined by matching the inner expansion with the proper outer expansion at some arbitrary interparticle distance from the test sphere.

Outer expansion. As the interparticle distance increases, the contribution of the interparticle potential to the radial flux decreases quite rapidly. In fact, for interparticle distances larger than approximately $\left(a_{1}+a_{2}\right) / G r$, the effect of interparticle interactions is negligible compared to the convection due to gravity (19). Consequently, in the outer expansion the interparticle potential has only a weak influence on the probability density, and can be well represented by (15)

$$
\frac{1}{k T} \frac{\partial V_{\mathrm{int}}}{\partial R}=\frac{K_{0}}{R^{3}},
$$

since at large interparticle distances $V_{\text {int }}$ $\propto R^{-2}(11)$, where $K_{0}$ is a constant. Substituting this expression and the far-field asymptotic expressions for $\mathcal{A}(R), \mathcal{B}(R), \mathcal{S}(R)$, and $\mathscr{H}(R)$ into [7], and introducing the new variable $\hat{R}$ defined by

$$
\hat{R}=G r R,
$$

yields, when one neglects terms of order $\mathrm{Gr}^{3}$,

$$
\begin{aligned}
\frac{\partial}{\partial \hat{R}}\left\{\left[\hat{R}^{2}-\xi G r \hat{R}\right] \frac{\partial \mathscr{P}}{\partial \hat{R}}+\frac{G r^{2} K_{0} \mathscr{P}}{\hat{R}}\right\} & \\
+ & {\left[1-\frac{\xi}{2 \hat{R}} G r\right] \nabla_{\theta}^{2} \mathscr{P} } \\
= & {\left[\hat{R}-\frac{\beta}{2} G r\right] \sin \theta \frac{\partial \mathscr{P}}{\partial \theta} } \\
& -\left[\hat{R}^{2}-\beta G r \hat{R}\right] \cos \theta \frac{\partial \mathscr{P}}{\partial \hat{R}} .
\end{aligned}
$$

One notices that, as $\mathrm{Gr} \rightarrow 0$, the convection and diffusion terms become of the same order of magnitude. Consequently, [51] is the proper outer expansion, valid at large interparticle distances from the test sphere.

Once again, letting

$$
\mathscr{P}(\hat{R}, \theta)=\hat{\mathscr{P}}_{0}+\operatorname{Gr} \hat{\mathcal{P}}_{1}+\cdots,
$$

and substituting this expansion into [51], gives for $\hat{\mathcal{P}}_{i}$

Journal of Colloid and Interface Science, Vol. 101, No. L, September 1984

$$
\begin{aligned}
& \frac{\partial}{\partial \hat{R}} \hat{R}^{2} \frac{\partial \hat{\mathcal{P}}_{i}}{\partial \hat{R}}+\nabla_{\theta}^{2} \hat{\mathcal{P}}_{i} \\
& =\hat{R} \sin \theta \frac{\partial \hat{\mathcal{P}}_{i}}{\partial \theta}-\hat{R}^{2} \cos \theta \frac{\partial \hat{P}_{i}}{\partial \hat{R}}
\end{aligned}
$$

or in cartesian coordinates

$$
\nabla^{2} \hat{\mathcal{P}}_{i}+\frac{\partial \hat{\mathcal{P}}_{i}}{\partial \hat{X}_{1}}=0
$$

where $i=0$ or 1 (note: gravity acts along the $\hat{X}_{1}$ axis). The solution of [53] has been given by Acrivos and Taylor (32) and, for $\hat{\mathfrak{P}}_{0}$, can be written as

$$
\hat{\mathcal{P}}_{0}=k_{0}+k_{1} F(\hat{R}, \theta)
$$

where $k_{0}$ and $k_{1}$ are constants of integration and

$$
F(\hat{R}, \theta)=\frac{1}{\hat{R}} \exp \left\{-\frac{\hat{R}}{2}(1+\cos \theta)\right\} .
$$

Expanding [56], one finds that near the origin $F(\hat{R} \rightarrow 0, \theta)$

$$
=\frac{1}{\hat{R}}-\frac{1}{2}(1+\cos \theta)+\cdots
$$

The term $k_{1} F(\hat{R}, \theta)$ in [55] leads to an inverse power of $\mathrm{Gr}$ unless $k_{1}=0$. From the boundary condition $\hat{\mathcal{P}}_{0}(\infty, \theta)=1$, it follows that $k_{0}=1$. Analogous to the solution for $\hat{\mathcal{P}}_{0}$, the solution for $\hat{\mathscr{P}}_{1}$ can be written as

$$
\hat{\mathfrak{P}}_{1}=k_{2}+k_{3} F(\hat{R}, \theta) \text {. }
$$

Since $\hat{P}_{1}(\infty, \theta)=0$ and $F(\infty, \theta)=0$, it follows that $k_{2}=0$. However, $k_{3}$ remains unknown.

Therefore, the outer expansion at small $\hat{R}$ is

$$
\begin{aligned}
\mathcal{P}(\hat{R} \rightarrow & 0, \theta)=1+G r k_{3} \\
& \times\left\{\frac{1}{\hat{R}}-\frac{1}{2}(1+\cos \theta)\right\}+\cdots,
\end{aligned}
$$

or

$$
\begin{aligned}
\mathscr{P}(R \rightarrow \infty, \theta) & =1+\frac{k_{3}}{R} \\
& -\operatorname{Gr}\left\{\frac{k_{3}}{2}(1+\cos \theta)\right\}+\cdots
\end{aligned}
$$


Only the first two terms of [57] are included in the outer expansion since the $\hat{R}, \hat{R}^{2}$, etc. terms will result in contributions of higher order than Gr.

Matching inner and outer expansions. As previously shown, the $f_{\ell}(R)$ portion of the inner expansion (see [40]) at large $R$ reduces to (15)

$$
f_{\ell}(R)=a_{\ell 1} R^{\ell}+\frac{a_{\ell 2}}{R^{\ell+1}} .
$$

In the first term of this solution $\ell$ can only be 0 or 1 , since $\ell \geqslant 2$ would require inverse powers of $\mathrm{Gr}$ in the outer expansion (see [50], [59], and [60]); this is impossible since these terms would approach infinity as $\mathrm{Gr} \longrightarrow 0$. Therefore, it follows that $a_{\ell 1}=0$ for $\ell \geqslant 2$. In addition, in the second term of [46] $\ell$ can only be 0 , since there are no $R^{-(\ell+1)}$ terms for $\ell \geqslant 1$ in the outer expansion (see [56] and [57]). Consequently, $a_{\ell 2}=0$ for $\ell \geqslant 1$. Therefore, the first order correction for gravity (in the inner region) at large $R$ reduces to

$$
\begin{aligned}
\mathscr{P}_{1}(R \rightarrow \infty, \theta)=\frac{\alpha_{\mathrm{Br}}}{2} & \cos \theta+a_{01} \\
& +\frac{a_{02}}{R}+a_{11} R \cos \theta,
\end{aligned}
$$

and the inner expansion at large $R$ becomes

$$
\begin{array}{r}
\mathscr{P}(R \rightarrow \infty, \theta)=1-\frac{\alpha_{\mathrm{Br}}}{R}+\operatorname{Gr}\left\{\frac{\alpha_{\mathrm{Br}}}{2} \cos \theta+a_{01}\right. \\
\left.+\frac{a_{02}}{R}+a_{11} R \cos \theta\right\}+\cdots \quad[62]
\end{array}
$$

Matching the inner expansion [62] at large $R$ with the outer expansion [60] at small $\hat{R}$ yields

$$
\begin{gathered}
k_{3}=-\alpha_{\mathrm{Br}} ; \\
a_{01}=-\frac{k_{3}}{2}=\frac{\alpha_{\mathrm{Br}}}{2} ; \text { and } a_{11}=0
\end{gathered}
$$

It should be noted that $a_{02}$ cannot be zero even though there is no $R^{-1}$ term explicitly shown in the outer expansion's first order correction $\hat{P}_{1}$ (see [60]). There are two reasons why $a_{02} \neq 0$ : (i) if $a_{02}=0$ then the inner boundary condition of [42] can not be satisfied; and (ii) the $R^{-1}$ term in the outer expansion actually comes from the second order correction $\hat{\mathcal{P}}_{2}$. The former point is discussed next, while the latter is covered in the Appendix.

The complete inner solution of order $G r$ is

$$
\mathcal{P}_{1}(R, \theta)=f_{p}(R) \cos \theta+f_{0}(R) .
$$

Similarly to the derivation of [35], one can show that (13)

$f_{0}=e^{-V_{\mathrm{int} / k T}}\left\{a_{01}+a_{02} \int_{R}^{\infty} \frac{e^{V_{\mathrm{in} t} / k T}}{S(R) R^{2}} d R\right\}$.

Since $f_{0}(2)=0$,

$a_{02}=-\frac{a_{01}}{\int_{2}^{\infty} \frac{e^{V_{\text {in } / k T}}}{S(R) R^{2}} d R}=-\alpha_{\mathrm{Br}} a_{01}=-\frac{\alpha_{\mathrm{Br}}^{2}}{2}$.

One notices that if $a_{02}=0$ then $a_{01}$ would have to be zero in order to satisfy the inner boundary condition; however, from the matching procedure it was determined that $a_{01}=\alpha_{\mathrm{Br}} / 2$, which indicates that $a_{02}$ cannot be zero.

\section{Solution of the Pair-Conservation Equation for Small Gr}

Substituting the values of the constants determined from the matching procedure into [62] gives the following steady-state solution of the pair-conservation equation, Eq. [7], valid at large $R$ :

$$
\begin{aligned}
\mathscr{P}=1- & \frac{\alpha_{\mathrm{Br}}}{R}+\frac{G r \alpha_{\mathrm{Br}}}{2} \\
& \times\left\{(1+\cos \theta)-\frac{\alpha_{\mathrm{Br}}}{R}\right\}+\cdots,
\end{aligned}
$$

when $\alpha_{\mathrm{Br}} \neq 0$. It should be noted that in the derivation of [65], the symmetry condition given by [11] was not invoked. However, it is easily shown that [65] does indeed satisfy this condition.

While [65] describes the first order effect of gravity on the particle distribution, the following question still remains: what is the magnitude of the next order term in [65]? An 
examination of [51] reveals that this equation is very similar to the outer expansion of the classic heat and mass transfer equation for single spheres in a Stokes velocity field (32); the major difference being the inclusion of the interparticle force term. Based on Acrivos and Taylor's (32) perturbation solution one can conclude that the next term in [65] will be of order $G r^{2} \ln G r$.

\section{FLOCCULATION RATES}

\section{Coupled Rates}

In this section the probability density given by [65] will be used to determine coupled gravity-induced/Brownian flocculation rates for small gravity numbers $\mathrm{Gr}$.

Choosing the area $A^{*}$ shown in Fig. 1 as a sphere with radius $r^{*}$, substituting the velocity expressions [4]-[6] into [1], and dedimensionalizing, gives

$$
\begin{aligned}
j_{12}= & \frac{D_{0}\left(a_{1}+a_{2}\right) N_{02}}{2} \\
& \times \int_{\theta=0}^{\pi} \int_{\phi=0}^{2 \pi}\left\{S(R)\left(\frac{\partial \mathcal{P}}{\partial R}+\frac{\mathcal{P}}{k T} \frac{\partial V_{\mathrm{int}}}{\partial R}\right)\right. \\
& +\operatorname{Gr} \mathcal{A}(R) \cos \theta \mathcal{P}\} R^{2} \sin \theta d \phi d \theta .
\end{aligned}
$$

The three terms under the integral sign represent the flux due to diffusion, interparticle forces, and gravitational forces. Substitution of the probability density function given by [65] into [66] yields for the coupled flocculation rate:

$$
\begin{aligned}
j_{12}=\frac{4 \pi D_{0}\left(a_{1}+a_{2}\right) N_{02}}{W_{\mathrm{Br}}} & \left(1+\frac{G r}{W_{\mathrm{Br}}}\right) \\
& +0\left(G r^{2} \ln \mathrm{Gr}\right) .
\end{aligned}
$$

The Brownian flocculation stability factor $W_{\mathrm{Br}}$ can be computed from [36] once the form of the total interparticle potential $V_{\text {int }}$ is known. The most useful formalization of this potential for colloidal systems is described by the well known DLVO theory $(27,28)$, which assumes additivity of the separate attractive and repulsive potentials.

Numerical values of $W_{\mathrm{Br}}$ are presented in Table I, which were computed using the interparticle potentials given in (18). The important dimensionless parameters are

$$
\begin{aligned}
N_{\mathrm{A}} & =\frac{2 A a_{1} a_{2}}{k T\left(a_{1}+a_{2}\right)^{2}}, \\
N_{\mathrm{R}} & =\frac{\epsilon \Psi_{0}^{2}\left(a_{1}+a_{2}\right)}{2 A}, \\
\kappa & =\frac{K\left(a_{1}+a_{2}\right)}{2},
\end{aligned}
$$

and

$$
\nu=\frac{2 \lambda_{\mathrm{L}}}{\left(a_{1}+a_{2}\right)},
$$

where $A$ is the Hamaker constant, $\epsilon$ the dielectric constant of the medium, $\Psi_{0}$ the surface

TABLE I

Sample Calculations of $W_{\mathrm{Br}}$ and $W_{\mathrm{Gr}}{ }^{a}$

\begin{tabular}{ccccccc}
\hline$G r$ & $\lambda$ & $\kappa$ & $N_{\mathrm{R}}$ & $N_{\mathrm{A}}$ & $W_{\text {Br }}$ & $W_{\mathrm{Gr}^{b}}$ \\
\hline $10^{-2}, 10^{-1}$ & 0.1 & 1300 & 400 & 0.19948 & 1.387 & $1.759,4.412$ \\
$10^{-2}, 10^{-1}$ & 0.2 & 1300 & 400 & 0.33524 & 1.635 & $1.210,3.197$ \\
$10^{-2}, 10^{-1}$ & 0.5 & 1300 & 400 & 0.53638 & 2.037 & $1.253,2.822$ \\
$10^{-2}, 10^{-1}$ & 0.9 & 1300 & 400 & 0.60176 & 2.186 & $1.249,3.500$ \\
$10^{-2}, 10^{-1}$ & 0.2 & 800 & 400 & 0.33524 & 3.283 & $1.210,3.197$ \\
$10^{-2}, 10^{-1}$ & 0.5 & 800 & 400 & 0.53638 & 17.73 & $1.253,2.822$ \\
$10^{-2}, 10^{-1}$ & 0.2 & 750 & 400 & 0.33524 & 19.13 & $1.210,3.197$ \\
$10^{-2}, 10^{-1}$ & 0.5 & 750 & 400 & 0.53638 & 703.4 & $1.253,2.822$ \\
\hline
\end{tabular}

${ }^{a}$ Parametric values: $A=5 \times 10^{-14}$ ergs, $\nu=0.1, T=300^{\circ} \mathrm{K}, \gamma=1$.

${ }^{b}$ For $W_{\mathrm{Gr}}$ the first number corresponds to $\mathrm{Gr}=10^{-2}$ and the second to $\mathrm{Gr}=10^{-1}$. 
potential, $K$ the reciprocal of the DebyeHückel double layer thickness, and $\lambda_{\mathrm{L}}$ the characteristic London wavelength of the atoms.

The percentage increase in the flocculation rate due to gravity is shown in Fig. 3 for various values of the particle size ratio $\lambda$. In order to remove the dependence of the gravity number $G r$ on $\lambda$, a normalized gravity number $G r^{*}$ is used, namely

$$
G r^{*}=\frac{G r}{\lambda\left(1-\lambda^{2}\right)}=\frac{2 \pi g \Delta \rho a_{2}^{4}}{3 k T} .
$$

It is evident from this figure that for the small gravitational forces considered here, gravity increases the flocculation rate for all particle size ratios (except for $\lambda=0$ and 1 , see [8]). In addition, the effect of gravity increases with increasing $\lambda$ and reaches a maximum at $\lambda$ $\simeq 0.58$ (see Fig. 2), afterwhich the effect of gravity decreases with increasing $\lambda$.

\section{Additivity vs Coupling}

In this section the total flocculation rate computed from the perturbation analysis will be compared with the old additivity assumption.

According to the theory of Brownian flocculation $(10,11)$ the net flux $\partial_{\mathrm{Br}}$ due to Brownian motion in the absence of any external forces is

$$
\mathscr{f}_{\mathrm{Br}}=\frac{4 \pi D_{0}\left(a_{1}+a_{2}\right) N_{02}}{W_{\mathrm{Br}}} .
$$

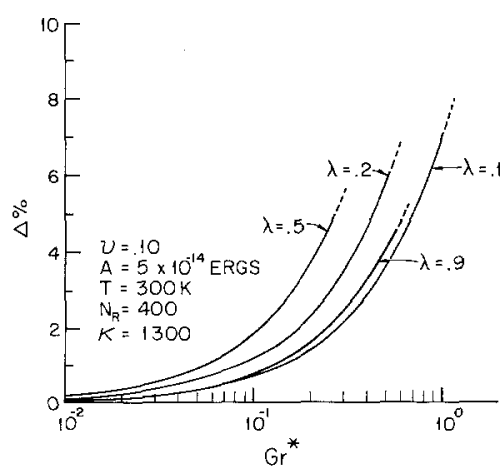

FIG. 3. Percentage increase in the flocculation rate due to gravity for various particle size ratios.
Saffman and Turner (33) have determined the net flux $\phi_{\mathrm{Gr}}$ due to pure gravitational motion as

$$
\partial_{\mathrm{Gr}}=\frac{\pi\left(u_{02}-u_{01}\right)\left(a_{1}+a_{2}\right)^{2} N_{02}}{W_{\mathrm{Gr}}}
$$

where $W_{G r}$ is the gravity-induced flocculation stability factor. In Saffman and Turner's original derivation both interparticle forces and hydrodynamic interactions were neglected and, consequently, the stability factor $W_{\mathrm{Gr}}$ is introduced into their result to account for these effects. Assuming additivity of the individual flocculation rates gives

$$
\begin{aligned}
j_{12} & =\partial_{\mathrm{Br}}+\partial_{\mathrm{Gr}} \\
& =\frac{4 \pi D_{0}\left(a_{1}+a_{2}\right) N_{02}}{W_{\mathrm{Br}}}\left(1+\frac{G r}{2} \frac{W_{\mathrm{Br}}}{W_{\mathrm{Gr}}}\right)
\end{aligned}
$$

Comparing [67] and [75], one notices that additivity is justified for $G r \ll 1$ only when $W_{\mathrm{Gr}} \simeq W_{\mathrm{Br}}^{2} / 2$.

The gravity-induced flocculation stability factor $W_{\mathrm{Gr}}$ can be computed from a trajectory analysis when the different sized spheres are subjected to interparticle, gravitational, as well as hydrodynamic forces, in the absence of Brownian motion. The complete details of this analysis are given elsewhere $(18,23)$; however, some numerical examples are given in Table I for various gravitational and interparticle strengths.

Figure 4 shows the magnitude of the percentage difference between the flocculation rates computed from [67] and [75] for various interparticle strengths. When electrostatic repulsion is negligible (curves 1 and 2), the additivity assumption gives results which are in fair agreement with the perturbation analysis. On the other hand, for cases in which electrostatic repulsion is moderately significant (curves 3-5), the two theories can give widely different results. For example, when $\lambda=0.2$ and $\kappa=750$ (curve 5), additivity overpredicts the total flocculation rate by as much as $24 \%$.

\section{DISCUSSION}

The effect of gravity on the total flocculation rate shown in Fig. 3 arises from the increased 


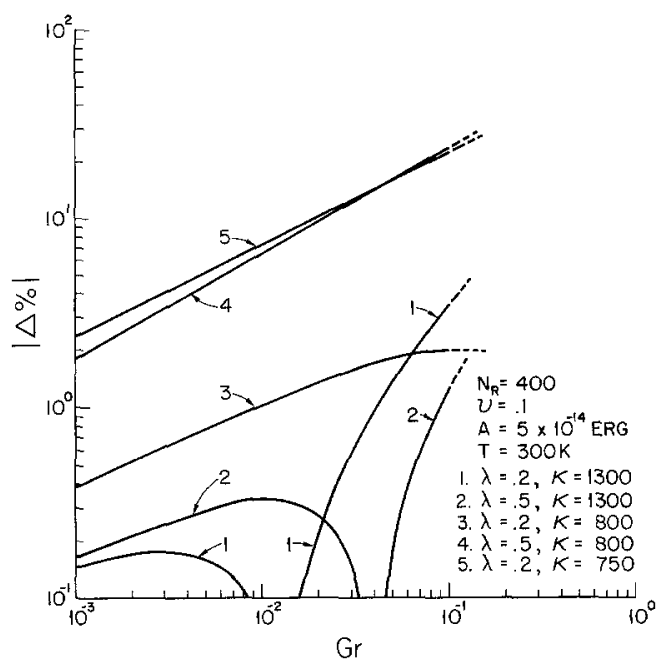

FIG. 4. Magnitude of the percentage difference between coupled flocculation rates and additive rates for various interparticle strengths.

probability of finding a type- 2 particle near the reference sphere. This increase in probability is reflected in [65]. However, while gravity can increase the probability density for a given interparticle separation, gravity does not significantly effect the actual flocculation process. This phenomenon is shown in [43], which indicates that the behavior of the total interparticle potential ultimately determines whether particle capture will occur.

Adding the individual Brownian and gravity-induced flocculation rates to obtain the total flocculation rate assumes that whatever mechanism is primarily responsible for bringing the particles into close proximity, either diffusion or differential creaming, is also responsible for overcoming the repulsive forces due to hydrodynamic and electric double layer interactions. However, the present analysis shows that the major influence of gravity on the flocculation process arises only from the outer expansion which is valid in a region where the particle trajectories follow the undisturbed fluid streamlines; this effect is reflected in Eq. [54]. Physically this means that gravity effects the particle distribution around the reference sphere at large interparticle distances only. This result indicates that while diffusion and differential creaming simultaneously bring the particles very close to each other, it is primarily diffusion and the van der Waals attractive force which eventually overcome the repulsive forces so that particle capture can occur.

\section{SUMMARY}

The singular perturbation technique has been used to solve the steady-state convectivediffusion equation for the case of strong Brownian/weak gravity effects. From this solution a simple analytical expression for coupled gravity-induced/Brownian flocculation rates has been obtained (see [67]). For the small gravitational forces considered in this paper, gravity enhances the flocculation rate with a maximum increase occuring for particle size ratios equal to approximately 0.58 .

In addition, these coupled flocculation rates were compared with the rates obtained by summing the individual flocculation rates due to Brownian motion and differential creaming. Under conditions of negligible electrostatic repulsion, both approaches yield similar results. However, when electrostatic repulsion is important, additivity can overpredict the total flocculation rate by as much as $24 \%$.

\section{APPENDIX: SECOND ORDER SOLUTION IN THE OUTER EXPANSION}

As discussed previously, if one were to strictly follow the matching procedure, this would lead to the erroneous conclusion that $a_{02}=0$ since there is no $R^{-1}$ term in the outer expansion's first order correction $\hat{\mathcal{P}}_{1}$ (see [60]). In this appendix it will be shown that the $R^{-1}$ term in $\hat{\mathscr{P}}_{1}$ originates in the second order correction $\hat{\mathcal{P}}_{2}$.

Substituting the expansion

$$
\begin{aligned}
\mathscr{P} & =\hat{\mathcal{P}}_{0}+G r \hat{\mathcal{P}}_{1}+G r^{2} \hat{\mathcal{P}}_{2} \\
& =1-\alpha_{\mathrm{Br}} G r F(\hat{R}, \theta)+G r^{2} \hat{\mathcal{P}}_{2}
\end{aligned}
$$


into [51], gives for $\hat{P}_{2}$

$$
\begin{gathered}
\frac{\partial}{\partial \hat{R}} \hat{R}^{2} \frac{\partial \hat{\mathcal{P}}_{2}}{\partial \hat{R}}+\nabla_{\theta}^{2} \hat{\mathcal{P}}_{2} \\
-\hat{R} \sin \theta \frac{\partial \hat{\mathcal{P}}_{2}}{\partial \theta}+\hat{R}^{2} \cos \theta \frac{\partial \hat{\mathcal{P}}_{2}}{\partial \hat{R}} \\
=-\alpha_{\mathrm{Br}} \xi \frac{\partial}{\partial \hat{R}} \hat{R} \frac{\partial F}{\partial \hat{R}}-\frac{\alpha_{\mathrm{Br}} \xi}{2 \hat{R}} \nabla_{\theta}^{2} F+\frac{1}{2} \alpha_{\mathrm{Br}} \beta \\
\quad \times \sin \theta \frac{\partial F}{\partial \theta}-\alpha_{\mathrm{Br}} \beta \hat{R} \cos \theta \frac{\partial F}{\partial \hat{R}}+\frac{K_{0}}{\hat{R}^{2}}
\end{gathered}
$$

Since [A2] is a linear differential equation, the complete solution is given by the sum of the homogeneous solution and a particular solution. Therefore, the complete solution of [A2] can be written as (see [53] and [55])

$$
\hat{\mathcal{P}}_{2}=k_{4}+k_{5} F(\hat{R}, \theta)+\hat{\mathcal{P}}_{2 p}
$$

where $\hat{\mathcal{P}}_{2 p}$ is the particular solution of [A2]. From the boundary condition $\hat{\mathcal{P}}_{2}(\infty, \theta)=0$, it follows that $k_{4}=0$ since $F(\infty, \theta)=0$ and $\hat{\mathcal{P}}_{2 p}(\infty, \theta)=0$. Substituting [57] into [A3] gives

$$
\begin{aligned}
\hat{\mathcal{P}}_{2}=k_{5}\left\{\frac{1}{\hat{R}}-\frac{1}{2}(1+\cos \theta)\right\} & \\
& +\hat{\mathcal{P}}_{2 p}(\hat{R}, \theta) .
\end{aligned}
$$

Therefore, the $0\left(G r^{2}\right)$ term in the outer expansion at small $\hat{R}$ is

$$
\begin{aligned}
& \operatorname{Gr}^{2} k_{5}\left\{\frac{1}{\hat{R}}-\frac{1}{2}(1+\cos \theta)\right\} \\
&+\operatorname{Gr}^{2} \hat{\mathcal{P}}_{2 p}(\hat{R}, \theta) .
\end{aligned}
$$

Making use of [50], [A5] becomes

$$
\begin{aligned}
\frac{G r k_{5}}{R}-G r^{2}\left\{\frac{k_{5}}{2}(1+\right. & \cos \theta)\} \\
& +G r^{2} \hat{\mathcal{P}}_{2 p}(\mathrm{GrR}, \theta) . \quad \text { [A6] }
\end{aligned}
$$

One notices that there is indeed a $R^{-1}$ term of order $G r$ which could easily be substituted into the outer expansion at small $\hat{R}$ (see [60]). From the matching procedure one finds that $k_{5}=a_{02}=-\alpha_{\mathrm{Br}}^{2} / 2$.
NOTATION

$A \quad$ Hamaker constant

$A^{*} \quad$ Arbitrary flux integration surface

$a_{i} \quad$ Particle radius

$a_{\ell i} \quad$ Constant of integration in the inner expansion

$D_{0} \quad$ Stokes-Einstein relative diffusion coefficient $=\left(k T / 6 \pi \gamma \mu_{\mathrm{f}}\right)\left(1 / a_{1}\right.$ $\left.+1 / a_{2}\right)$

$\mathbf{e}_{\mathrm{r}}, \mathbf{e}_{\theta}, \mathbf{e}_{\phi} \quad$ Unit vectors in spherical polar coordinates

$F(\hat{R}, \theta) \quad$ Function defined in [56]

$f_{\ell}(R) \quad$ Function representing the radial portion of the homogeneous solution for $\mathcal{P}_{i}$

$f_{p}(R) \quad$ Function representing the radial portion of the particular solution of $\mathscr{P}_{1}$

Local acceleration of gravity

Dimensionless interparticle separation $=R-2$

Single-particle flocculation rate

Constant defined in [49]

Constant of integration in the outer expansion

$k \quad$ The Boltzmann constant

$L_{\ell}(\cos \theta)$ Legendre polynomial of the first kind

$N_{02} \quad$ Bulk concentration of particles of radius $a_{2}$

n Outward unit normal

$R$ Dimensionless interparticle distance $=2 r /\left(a_{1}+a_{2}\right)$

$\hat{R}=$ GrR

Position vector relative to the test sphere

$\begin{array}{ll}r & \text { Distance between particle centers } \\ r^{*} & \text { Radius of a sphere with a surface }\end{array}$ area $A^{*}$

$T \quad$ Absolute temperature

$u_{0 i} \quad$ Stokes creaming rate for particles of size $a_{i}=2 g \Delta \rho a_{i}^{2} / 9 \gamma \mu_{\mathrm{f}}$

$V_{\text {int }} \quad$ Total interparticle potential

v Relative sphere velocity

$\mathbf{v}_{\mathrm{Br}} \quad$ Relative sphere velocity due to Brownian motion 


\begin{tabular}{|c|c|}
\hline$v_{g}$ & $\begin{array}{l}\text { Relative sphere velocity due to } \\
\text { gravity }\end{array}$ \\
\hline int & $\begin{array}{l}\text { Relative sphere velocity due to in- } \\
\text { terparticle forces }\end{array}$ \\
\hline$W_{\mathrm{Br}}$ & $\begin{array}{l}\text { Brownian flocculation stability } \\
\text { factor }\end{array}$ \\
\hline$W_{\mathrm{Gr}}$ & $\begin{array}{l}\text { Gravity-induced flocculation sta- } \\
\text { bility factor }\end{array}$ \\
\hline $\mathcal{A}(R)$ & $\begin{array}{l}\text { Hydrodynamic correction to } \\
\text { Stokes relative creaming rate } \\
\left(u_{02}-u_{01}\right) \text { for particles moving } \\
\text { parallel to their line of centers }\end{array}$ \\
\hline $\mathscr{B}(R)$ & $\begin{array}{l}\text { Hydrodynamic correction to } \\
\text { Stokes relative creaming rate } \\
\left(u_{02}-u_{01}\right) \text { for particles moving } \\
\text { perpendicular to their line of } \\
\text { centers }\end{array}$ \\
\hline$g_{(R)}$ & $\begin{array}{l}\text { Hydrodynamic correction to } \\
\text { Stokes-Einstein relative diffu- } \\
\text { sion coefficient } D_{0} \text { for particles } \\
\text { moving parallel to their line of } \\
\text { centers }\end{array}$ \\
\hline $\mathscr{Z}(R)$ & $\begin{array}{l}\text { Hydrodynamic correction to } \\
\text { Stokes-Einstein relative diffu- } \\
\text { sion coefficient } D_{0} \text { for particles }\end{array}$ \\
\hline
\end{tabular}

to London van der Waal attractive forces

Brownian capture efficiency defined in [36]

Constant defined in [17]

Viscosity ratio; defined in [19]

Azimuthal coordinate-spherical polar coordinate system

Dimensionless reciprocal of the double layer thickness

Particle size ratio $=a_{1} / a_{2}$

Viscosity of the suspending fluid

Viscosity of the suspended particles

Dimensionless electromagnetic retardation parameter

Density difference between the particles and the suspending fluid

Polar coordinate-spherical polar coordinate system

Constant defined in [18]

The "del" operator

Laplacian operator

Radial operator defined in [30]

Tangential operator defined in [31]

REFERENCES

$\delta_{\mathrm{Br}} \quad$ Net-flux due to Brownian motion

$\delta_{\mathrm{Gr}} \quad$ Net-flux due to gravity

$\ell \quad$ An integer

$\mathcal{P} \quad$ Probability density

$\mathscr{P}_{i} \quad$ ith order contribution to $\mathscr{P}$

$\hat{\mathcal{P}} \quad$ Probability density in the outer expansion

$\hat{\mathcal{P}}_{i} \quad i$ th order contribution to $\hat{\mathcal{P}}$

$\mathrm{Gr} \quad$ Gravity number; represents the relative importance of gravitational to thermal (Brownian) forces

$N_{\mathrm{A}} \quad$ Dimensionless number describing the relative importance of London van der Waal attractive forces to the system thermal forces

$N_{\mathrm{R}} \quad$ Dimensionless number describing the relative importance of electric double layer repulsive forces

1. Reddy, S. R., Melik, D. H., and Fogler, H. S., J. Colloid Interface Sci. 82, 116 (1981).

2. Reddy, S. R., and Fogler, H. S., J. Colloid Interface Sci. 82, 128 (1981).

3. Müller, V. H., Kolloidchem. Beih. 27, 223 (1928); for an English translation of Müller's derivation see: Matthews, B. A., J. Pharm. Sci. 62, 172 (1973).

4. Von Smoluchowski, M., Z. Phys. Chem. 92, 129 (1917).

5. Matthews, B. A., and Rhodes, C. T., J. Colloid Interface Sci. 32, 332 (1970).

6. Prieve, D. C., and Lin, M. J., J. Colloid Interface Sci. 76, 32 (1980).

7. Adamczyk, Z., and van de Ven, T. G. M., J. Colloid Interface Sci. 80, 340 (1980).

8. Rajagopalan, R., and Kim, J. S., J. Colloid Interface Sci. 83, 428 (1981).

9. Adamczyk, Z., and van de Ven, T. G. M., J. Colloid Interface Sci. 84, 497 (1981).

10. Derjaguin, B. V., and Muller, V. M., Dokl. Akad. Nauk. S. S. S. R. (English Translation), 176, 738 (1967).

11. Spielman, L. A., J. Colloid Interface Sci. 33, 562 (1970). 
12. Fuchs, N., Z. Phys. 89, 736 (1934).

13. Roebersen, G. J., and Wiersema, P. H., J. Colloid Interface Sci. 49, 98 (1974).

14. Feke, D. L., and Prabhu, N., Presented at the AIChE 1982 Annual Meeting, Los Angeles, Ca. (Nov. 1419).

15. van de Ven, T. G. M., and Mason, S. G., Colloid Polym. Sci. 255, 794 (1977).

16. Batchelor, G. K., J. Fluid Mech. 83, 97 (1977).

17. Feke, D. L., and Schowalter, W. R., J. Fluid Mech., 133, 17 (1983); see also: Feke, D. L., Ph.D. Thesis, Princeton University (1981).

18. Melik, D. H., and Fogler, H. S., J. Colloid Interface Sci. 101, 72 (1984).

19. Batchelor, G. K., J. Fluid Mech. 119, 379 (1982).

20. Batchelor, G. K., and Wen, C-S., J. Fluid Mech. 124, 495 (1982).

21. Proudman, I., and Pearson, J. R. A., J. Fluid Mech. 2, 237 (1957).

22. Happel, J., and Brenner, H., "Low Reynolds Number Hydrodynamics." Noordhoff International Publishing, Leyden, The Netherlands, 1965.

23. Melik, D. H., Ph.D. Thesis, University of Michigan (1984).

24. Batchelor, G. K., J. Fluid Mech. 74, 1 (1976).
25. Reed, C. C., and Anderson, J. L., AIChE J. 26, 816 (1980).

26. van de Ven, T. G. M., and Mason, S. G., J. Colloid Interface Sci. 57, 505 (1976).

27. Derjaguin, B., and Landau, L., Acta Physiochim. 14, 633 (1941).

28. Verweey, E. J. W., and Overbeek, J. Th. G., "Theory of the Stability of Lyophobic Colloids." Elsevier, Amsterdam, 1948.

29. Ruckenstein, E., J. Colloid Interface Sci. 66, 531 (1978).

30. Prieve, D. C., and Ruckenstein, E., J. Colloid Interface Sci. 73, 539 (1980).

31. Bacon, J., Dickinson, E., Parker, R., Anastasiou, N., and Lal, M., J. Chem. Soc., Faraday Trans. 2 79, 91 (1983).

32. Acrivos, A., and Taylor, T. D., Phys. Fluids 5, 387 (1962).

33. Saffman, P. F., and Turner, J. S., J. Fluid Mech. 1, 16 (1956).

34. Adamczyk, Z., and van de Ven, T. G. M., J. Colloid Interface Sci. 97, 68 (1984).

35. Adamczyk, Z., Dabroś, T., Gzarnecki, J., and van de Ven, T. G. M., J. Colloid Interface Sci. 97, 91 (1984).

36. Dickinson, E., and Parker, R., J. Colloid Interface Sci. 97, 220 (1984). 